\title{
Review of: "SARS-CoV-2 infects human adipose tissue and elicits an inflammatory response consistent with severe COVID-19"
}

Shie-Liang Hsieh

Potential competing interests: The author(s) declared that no potential competing interests exist.

In this manuscript, author apply single cell Seq and high dimensional FACS to analyze human adipose tissues-mediated inflammation during SARS-COV-2 infection. Author identify mature adipocytes and adipose tissue macrophages new targets for SARS-COV-2. Author detect SARS-CoV-2 RNA in adipocytes, while SARS-CoV-2 is restricted to a highly inflammatory subpopulation of macrophages. Author claims that this finding can explain the link between obesity and severe OVID-19.

General comments:

In general, this is a very interesting and important work regarding the pathogenesis of COVID-19. Even though the evidence regarding adipose tissue in SARS-COV-2 infection is supported by their data, but several key issues need to be further clarified.

1) what is the receptor for virus entry to adipose tissue (adipocyte)?

2) How SARS-CoV-2 activates adipose tissue macrophages? How to distinguish infiltrating macrophages from resident adipose tissue macrophages?

Specific comments

1. Figure 1D:

author claims SARS-CoV-2 N protein is detected in adipose macrophages. Author should display the percentage of macrophages (CD45+CD14+CD11c- cells) expressed N-protein. Are all this population infected by SARS-CoV-2, or only a limited percentage? what is the percentage of infected adipose tissue macrophage isolated from SAT, VAT?

1. Figure $2 \mathrm{E} \& 2 \mathrm{~F}$

Since author identify cluster 2 and cluster 12 are highly susceptible to SARS-CoV-2, author should pick up several surface markers differentially expressed in cluster 2 and cluster 4 to distinguish these two groups. 1. Figure 3A:

This figure is confusing because author put genes expressed in both cluster 2 and cluster 12 in a single panel. Author should split into two volcano plots to display genes up- and down-regulated in each cluster.

1. Figure 3C: The labeling is hard to understand. It looks like that the author get samples from three 
different individuals (participants). The left 3 columns are infected, while the right 3 columns are bystanders. In this case, the gene expression profile among the three participants are very different. If the basel level is very different among these three individuals, author should provide another figure to illustrate the relative fold change of each gene in infected vs bystander macrophages.

2. Figure 3D:

Author emphasize IL-10 pathways in SARS-CoV-2-infected C2 cluster cells, but does not mention the prominent enrichment chemokine pathway. This interpretation is misleading because:

a) In Table I, chemokines are the most dominant cytokines in SARS-CoV-2-infected SVC.

b) IL-10 is regarded as anti-inflammatory cytokines. If author claims adipose macrophages are responsible for disease severity, author should find upregulation of TNF, IL-6, and IL-1 cytokines.

1. Figure 4:

Author finds some ISGs, including ISG15, and IFIT1, and IFI127 were upregulated in preadipocytes. As these genes are critical in host defense against viral infections, suggesting adipose tissues are one of the major tissues secreting anti-viral cytokines, rather than proinflammatory cytokines. This argument is supported by the heat map (Fig 4F).

\section{Minor comments}

1) The manuscript is poor organized. The figures are inserted within text and very difficult to follow.

2) The resolution of figure is poor. PI should increase the quality of figure in the revised version. 